\title{
Original
}

\section{Influencia de la radioterapia sobre la reconstrucción mamaria inmediata posmastectomía ahorradora de piel. ¿Afecta igual antes que después?}

\author{
Marta Allué Cabañuz ${ }^{a, *}$, Maria Dolores Arribas del Amo ${ }^{a}$, Ana Navarro Barlés ${ }^{a}$ \\ y Antonio Tomás Guemes Sanchez ${ }^{b}$ \\ ${ }^{a}$ Unidad de Mama, Hospital Clínico Universitario Lozano Blesa, Zaragoza, España \\ ${ }^{\mathrm{b}}$ Profesor titular, Universidad de Zaragoza, jefe Unidad de Mama, Hospital Clínico Universitario Lozano Blesa, Zaragoza, España
}

\section{INFORMACIÓN DEL ARTÍCULO}

Historia del artículo:

Recibido el 25 de septiembre de 2018 Aceptado el 26 de octubre de 2018

On-line el $\mathrm{xxx}$

Palabras clave:

Radioterapia

Reconstrucción mamaria inmediata Implante directo

Mastectomía ahorradora de piel

Morbilidad a largo plazo

\begin{abstract}
R E S U M E N
Introducción: La mastectomía bilateral con reconstrucción inmediata (MB + RMI) está aumentando. La radioterapia incrementa las complicaciones, pero se han ampliado los criterios de administración. Queremos evaluar las tasas de complicaciones/secuelas realizando un análisis comparativo con una cohorte sin radioterapia.

Métodos: Análisis observacional analítico de cohortes retrospectivo de pacientes tratadas mediante MB como tratamiento de cáncer de mama con RMI entre 2000 y 2016. Se evalúan 3 grupos: grupo 1: pacientes previamente tratadas con cirugía local y radioterapia, y MB + RMI posterior; grupo 2: pacientes con $\mathrm{MB}+\mathrm{RMI}$ y radioterapia posterior por un cáncer de novo, y grupo 3: pacientes con $\mathrm{MB}+\mathrm{RMI}$ sin radioterapia previa ni posterior.

Evaluamos variables demográficas, técnicas quirúrgicas y morbilidad postoperatoria. Resultados: Se intervinieron un total de $296 \mathrm{MB}+\mathrm{RMI}$.

Grupo 1: 125 pacientes con radioterapia previa, administrada 21,69 meses antes de media. Tasa de complicaciones del $28,8 \%$, secuelas $33,6 \%$ y reintervención $33,6 \%$.

Grupo 2:71 pacientes con radioterapia tras reconstrucción 134,2 días de media. Tasa de complicaciones del $29,6 \%$, secuelas $19,9 \%$ y reintervención $16,9 \%$.

Grupo 3: 100 pacientes. Tasa de complicaciones del 30\%, secuelas 21\% y reintervención $20 \%$.

Encontramos más secuelas en el grupo 1, con casi el doble de reintervenciones que en el grupo 2 (33,6\% vs. $16,9 \%$; $p=0,067)$.

Conclusiones: La tasa de complicaciones a largo plazo y la tasa de reintervenciones es mayor en el grupo MB + RMI con radioterapia previa que en los grupos MB + RMI con radioterapia posterior $\mathrm{O} M B+\mathrm{RMI}$ sin radioterapia.
\end{abstract}

(C) 2018 Publicado por Elsevier España, S.L.U. en nombre de AEC.

\footnotetext{
* Autor para correspondencia.

Correo electrónico: martitaallue@hotmail.com (M. Allué Cabañuz). https://doi.org/10.1016/j.ciresp.2018.10.014

0009-739X/C 2018 Publicado por Elsevier España, S.L.U. en nombre de AEC.
} 
Keywords:

Radiotherapy

Immediate breast reconstruction

Direct to implant

Skin-sparing mastectomy

Long-term morbidity
Influence of radiotherapy on immediate breast reconstruction after skinsparing mastectomy. Before or after: Does it matter?

\section{A B S T R A C T}

Introduction: The use of bilateral mastectomy with immediate reconstruction is increasing. Radiotherapy increases complications; however, its uses have been extended. We evaluate the profile of the complications and long-term failure of reconstruction through a comparative analysis with a cohort without radiotherapy.

Methods: Retrospective analysis of patients with breast cancer who underwent mastectomy with immediate reconstruction during 2000-2016. Three groups were evaluated: 1) patients who received radiotherapy and posterior breast reconstruction; 2) patients with bilateral mastectomy and immediate reconstruction following adjuvant radiotherapy; 3) patients who did not receive radiotherapy at all. Demographic variables, surgical techniques and postoperative morbidity were assessed.

Outcomes and complications were compared between cohorts. Analysis was done with SPSS Statistics.

Results: 296 bilateral mastectomies with immediate reconstruction. Mean age $48.4 \pm 9$. No differences in comorbidity in the different groups.

Group 1: 125 patients. Radiotherapy given 21.69 months before, on average. Complication rate: $20 \%$. Failure of reconstruction rate: $20 \%$. Reoperation rate: $33.6 \%$.

Group 2: 71 patients. Radiotherapy after reconstruction: mean 134.2 days. Complication rate: $36.7 \%$. Failure of reconstruction rate: $21.1 \%$. Reoperation rate: $16.9 \%$.

Group 3: 100 patients. Complication rate: $25 \%$. Failure of reconstruction rate: $21 \%$. Reoperation rate: $20 \%$.

Morbidity published in patients after radiotherapy before or after reconstruction is higher than complications in patients who did not receive radiotherapy. Even so, in our series they were similar.

We found a higher sequelae rate in group 1, with almost double the rate of reoperation. Conclusions: Patients who underwent radiotherapy before reconstruction had a higher risk of developing failure of reconstruction and needing reoperation than those patients who received radiotherapy after breast reconstruction or did not receive radiotherapy at all.

(C) 2018 Published by Elsevier España, S.L.U. on behalf of AEC.

\section{Introducción}

A pesar de los avances terapéuticos, cerca del $45 \%$ de los pacientes con cáncer de mama serán tratadas mediante mastectomía $^{1}$ y hasta un $20-40 \%$ asociará alguna técnica reconstructiva con el objetivo de mejorar la calidad de vida y disminuir el impacto sociopsicológico de la mastectomía ${ }^{2}$.

En la última década, la mastectomía bilateral está experimentando un aumento y también las tasas de reconstrucción ${ }^{3}$, que puede ser diferida o inmediata, contando en este caso con un riesgo aumentado de complicaciones postoperatorias.

La radioterapia ha demostrado aumentar las tasas de complicación incluyendo infección, necrosis cutánea, contractura capsular, asimetría y la pérdida del implante ${ }^{4}$; sin embargo, los criterios para realizar mastectomías ahorradoras de piel que permitan una reconstrucción inmediata se han ido ampliando y parece seguro realizarlo incluso en pacientes sometidas previamente a radiación como parte del tratamiento conservador del cáncer de mama o las que precisan radioterapia adyuvante posmastectomía ${ }^{5,6}$.

El impacto de la radioterapia previa o administrada tras mastectomía con reconstrucción inmediata sigue siendo infrecuentemente informado.
El objetivo de este estudio es evaluar las tasas de complicaciones, secuelas y de reintervención y realizar un análisis comparativo con una cohorte de pacientes tratadas mediante mastectomía y reconstrucción inmediata sin asociar radioterapia.

Se pretende conocer aspectos específicos en esta población de pacientes para mejorar la calidad del asesoramiento preoperatorio y anticipar posibles complicaciones precoces como en el seguimiento a largo plazo.

\section{Métodos}

Análisis observacional analítico de cohortes retrospectivo de pacientes tratadas mediante mastectomía bilateral como tratamiento de un cáncer de mama con reconstrucción inmediata mediante prótesis directa en nuestro hospital durante el periodo 2000-2016. Se identificó a las pacientes tratadas mediante radiación previa tras cirugía conservadora y reconstrucción posterior, y a las pacientes con mastectomía y reconstrucción inmediata que precisaron radioterapia posteriormente. Asimismo, seleccionamos una cohorte de pacientes tratadas mediante mastectomía y reconstrucción inmediata sin tratamiento radioterápico previo ni posterior. 
Los criterios de inclusión para el grupo de pacientes con diagnóstico de recidiva local o nuevo tumor primario (grupo 1): las indicaciones fueron las anteriores y las siguientes:

- Deseo de la paciente de completar la mastectomía tras una cirugía conservadora primaria.

- Imposibilidad de completar radioterapia tras cirugía conservadora y radioterapia previa.

- Necesidad de simetrización contralateral.

Y para las pacientes con un cáncer de mama de novo (grupo 2 y grupo 3) fueron:

- Carcinoma multicéntrico o multifocal no susceptible de cirugía conservadora. Multifocalidad se define como la presencia de 2 o más focos tumorales en un mismo cuadrante y a menos de 5 $\mathrm{cm}$ del foco primario, y multicentricidad como la presencia de 2 o más focos tumorales en distintos cuadrantes de la misma mama o a más de $5 \mathrm{~cm}$ del foco primario.

- Gran componente in situ del tumor infiltrante encontrado en la biopsia diagnóstica preoperatoria.

- Alto riesgo por historia familiar (sin mutación conocida). Definido por 2 o más familiares (al menos uno de ellos de primer grado afectados de cáncer de mama u ovario a edades tempranas, antes de los 50 años).

- Mutación conocida en genes BRCA 1y2 u otras mutaciones responsables del incremento del riesgo.

Y se excluyó a las pacientes con edad avanzada (más de 70 años) y carcinoma inflamatorio, así como las MBRI sin enfermedad oncológica presente o pasada (mastectomía profiláctica pura).

Se recopilaron datos sobre variables demográficas (edad, comorbilidades como obesidad, hipertensión, diabetes mellitus, vasculopatías y tabaquismo activo de más de 10 cigarrillos al día, antecedentes personales y familiares), clínico-patológicas (indicación, tamaño tumoral y estado de la axila, etc.) y con relación al tratamiento quirúrgico y adyuvante; incluyendo el tipo de intervención quirúrgica, las diferentes técnicas reconstructivas empleadas y la morbilidad postoperatoria. Se evaluaron las complicaciones postoperatorias (aquellas que aparecieron dentro de los 30 días tras la intervención) y secuelas (pasados 30 días).

La técnica quirúrgica utilizada fue similar en todas las pacientes, resección del tejido mamario, dejando finos colgajos de piel, variando la incisión cutánea y la preservación completa de complejo aréola-pezón (CAP) o utilizando un injerto libre de CAP. Las incisiones varían según el tamaño y la configuración de la mama afectada y la contralateral, el tamaño y la localización del tumor, las cicatrices previas y la preferencia del cirujano, y deben planearse con vistas a la reconstrucción mamaria inmediata.

- Mastectomía subcutánea por incisión lateral externa. Técnica de Spira modificada: prótesis con doble cubierta mediante un colgajo desepitelizado unido al músculo pectoral mayor e injerto libre del CAP tras biopsia intraoperatoria negativa de la base del pezón.

- Mastectomía ahorradora de piel según patrón de Wise corto: se trata de un patrón típico de mamoplastia de reducción donde, además de la incisión periareolar, hay una prolongación vertical hacia el surco submamario con una extensión lateral y medial a lo largo del surco. De elección en mamas hipertrofiadas y ptósicas.

- Mastectomía ahorradora de piel y pezón por incisión radial externa. La reconstrucción inmediata se realizó mediante implante directo de silicona de perfil anatómico en la mayoría de pacientes 290/296 (98\%), exceptuando 6 casos en que se utilizó colgajo miocutáneo sin prótesis.

- Reconstrucción mediante tejido autógeno (con o sin prótesis asociada). Se emplearon 2 técnicas, utilizando en la mayoría de casos el colgajo de lattisimus dorsi y en 2 casos colgajo de músculo recto abdominal.

Los criterios para considerar la necesidad de tratamiento adyuvante con radioterapia siguieron las guías y las recomendaciones actualizadas en el momento del tratamiento de estas pacientes, así como las pautas y las duraciones de los mismos, teniendo en cuenta que han variado durante los años del estudio ${ }^{7-9}$. En las pacientes tratadas mediante mastectomía ahorradora de piel con RMI, las indicaciones han sido las mismas que tras una mastectomía estándar, es decir, irradiación de pared costal en tumores de gran tamaño T3T4, márgenes afectados o próximos, carcinoma in situ si hay margen afectado que no puede ser ampliado. Irradiación de pared costal y cadenas axiloclavicular si hay más de un ganglio afecto, tumores T4, e individualizando los casos en tumores G3, infiltración linfovascular, Her2 (+) o triple negativo.

\section{Análisis estadístico}

Para hallar diferencias significativas en las variables categóricas se emplearon los test estadísticos de la chi al cuadrado o de Fisher. Los test de Mann-Whitney y de la $t$ de Student fueron usados para variables no paramétricas y paramétricas, respectivamente. Un valor de $\mathrm{p}$ de 0,05 se consideró estadísticamente significativo. Análisis realizado con SPSS Statistics versión 22 .

\section{Resultados}

Durante el periodo de estudio se realizaron 296 intervenciones de mastectomía bilateral con reconstrucción inmediata $(\mathrm{MB}+\mathrm{RMI})$ como tratamiento de un cáncer de mama en nuestro centro. De estas, 125 pacientes $(42,2 \%)$ habían sido tratadas previamente con cirugía conservadora y radioterapia, y en un segundo tiempo se realizó mastectomía bilateral (totalización de la mastectomía y mastectomía contralateral reductora de riesgo) por diferentes motivos: márgenes de resección afectados o próximos, elección de la mastectomía en lugar de ampliación de márgenes por parte de la paciente, recidiva local, o por razones cosméticas para conseguir simetrización.

Otras 171 pacientes se intervinieron en un solo tiempo $(57,8 \%)$, es decir, la MB + RMI fue el tratamiento primario del cáncer de mama.

En este grupo, 71 pacientes precisaron radioterapia adyuvante. Las 100 pacientes restantes, que no precisaron radioterapia, se incluyen como la cohorte control (fig. 1). 


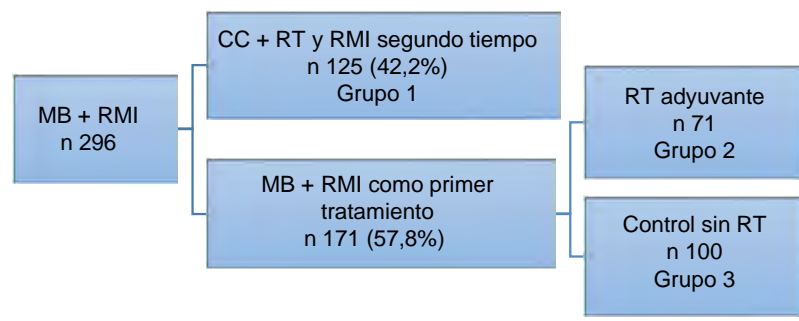

Figura 1 - Distribución de las pacientes en nuestra serie.

Resultados generales: las pacientes presentaban una edad media de 48,4 \pm 9,0 años (rango 26-87) en el momento de la intervención quirúrgica.

La indicación para practicar una MBRI más frecuente es la elección de la paciente, los márgenes de resección afectados o próximos seguidos de obtención de simetrización en el grupo de cáncer anterior (grupo 1) y multifocalidad seguida de elección de la paciente en el grupo de cáncer de novo (grupos 2 y 3), como se expone en la tabla 1.

Las comorbilidades que pudiesen afectar a la técnica quirúrgica y el desarrollo de complicaciones se exponen en la tabla 2. La distribución de estos factores entre los 3 grupos del estudio fue homogénea ( $p=0,017)$.

Técnicas quirúrgicas: en algunas pacientes se utilizaron técnicas de reconstrucción diferentes para cada mama, por ejemplo, un colgajo en la mama intervenida previamente (p. ej., tras mastectomía simple unilateral) y una mastectomía subcutánea en la mama sana contralateral (mastectomía profiláctica contralateral), mientras en otras pacientes se practicó una reconstrucción simétrica, por ejemplo, con la técnica de Spira. Los porcentajes se obtienen sobre el total de 592 procedimientos.

\section{Tabla 1 - Indicaciones MB + RMI en los diferentes grupos}

\begin{tabular}{lll} 
Indicaciones en pacientes con cáncer & $\mathrm{N}=171$ & $\%$ \\
de novo & & \\
\hline Cáncer bilateral & 13 & 7,6 \\
Cáncer unilateral & 158 & 92,4 \\
$\quad$ Multifocalidad & 47 & 27,4 \\
$\quad$ Elección de la paciente & 41 & 23,9 \\
$\quad$ Carcinoma lobulillar infiltrante (elección & 24 & 14,1 \\
de la paciente) & & \\
$\quad$ Multicentricidad & 20 & 11,7 \\
Carcinoma ductal in situ extenso & 13 & 7,6 \\
$\quad$ Elevado riesgo familiar sin mutación & 7 & 4,1 \\
conocida & 6 & 3,5 \\
$\quad$ Mutaciones de los genes BRCA1 y 2 & $\mathrm{N}=125$ & $\%$ \\
& & \\
Indicaciones para completar la & & \\
mastectomía por cáncer anterior & 44 & 35,2 \\
ya tratado & 32 & 25,6 \\
\hline Elección de la paciente & 23 & 18,4 \\
Márgenes de resección afectos o próximos & 16 & 12,8 \\
Obtención de simetrización & 4 & 3,2 \\
Tratamiento de la recidiva local & 3 & 2,4 \\
Cáncer contralateral & 3 & 2,4 \\
Mamas densas de difícil seguimiento & & \\
Mutaciones genes BRCA 1 y 2 detectadas a posteriori & 3 & \\
\hline & & \\
\hline
\end{tabular}

Tabla 2 - Distribución de factores de riesgo en los 3 grupos

\begin{tabular}{lllll} 
Comorbilidades & Total & Grupo 1 & Grupo 2 & Grupo 3 \\
\hline DM tipo 2 & $2,4 \%$ & $4 \%$ & $1,4 \%$ & $2 \%$ \\
$\begin{array}{l}\text { Obesidad en diferente } \\
\text { grado }\end{array}$ & $2,4 \%$ & $2,4 \%$ & $2,8 \%$ & $2 \%$ \\
HTA & $11,5 \%$ & $12,8 \%$ & $9,8 \%$ & $11 \%$ \\
Tabaquismo activo & $11,8 \%$ & $12 \%$ & $15,4 \%$ & $9 \%$ \\
\hline \multicolumn{4}{l}{ DM: diabetes mellitus; HTA: hipertensión arterial. } \\
\hline
\end{tabular}

La distribución de los diferentes tipos de reconstrucción en los 3 grupos del estudio fue homogénea, atendiendo a razones puramente técnicas, individualizando en cada paciente y eligiendo la técnica más idónea. No se encontraron diferencias estadísticamente significativas.

Presentamos los resultados de las diferentes técnicas utilizadas en la tabla 3.

Resultados grupo 1. Pacientes con cirugía conservadora y radioterapia previa a la mastectomía con reconstrucción: desde la radioterapia previa a la $\mathrm{MB}+\mathrm{RMI}$ pasó una media de 21,69 meses (rango 3-180 meses) en este grupo de pacientes.

La tasa general de complicaciones postoperatorias fue del $28,8 \%$ (36 de 125 casos) y de secuelas y mal resultado cosmético a largo plazo del 33,6\% (42/125), con una tasa de reintervención pasado el primer mes postoperatorio del 33,6\% (42/125). Los resultados se exponen en la tabla 4.

Resultados grupo 2. Pacientes con reconstrucción inmediata y radioterapia adyuvante: se realizó radioterapia adyuvante tras mastectomía con reconstrucción con una media de 134,2 \pm 126,9 días tras la intervención (rango 19-344 días).

La tasa general de complicaciones postoperatorias en fue del 29,6\% (21 de 71 casos) y de secuelas y mal resultado cosmético a largo plazo del 16,9\% (12/71); la tasa de reintervención pasado el primer mes postoperatorio fue de 16,9\% (12/71). Los resultados se exponen en la tabla 4.

Resultados del grupo 3. Cohorte control. Pacientes con mastectomía y reconstrucción inmediata sin radioterapia: las pacientes tratadas mediante mastectomía bilateral y reconstrucción sin indicación de radioterapia adyuvante presentaron una tasa general de complicaciones del 30\% (30/100) y de secuelas del $21 \%(21 / 100)$, y la tasa de reintervención pasado el

\section{Tabla 3 - Técnicas quirúrgicas realizadas y tasa de complicaciones/secuelas para cada técnica}

\begin{tabular}{llll}
\hline Técnica reconstructiva & B & $\%$ & $\begin{array}{c}\text { Tasa } \\
\text { complicaciones } \\
\text { y secuelas }\end{array}$ \\
\hline $\begin{array}{l}\text { Colgajos miocutáneos } \\
\quad \text { Con prótesis }\end{array}$ & 96 & 16,2 & $21,9 \%$ \\
$\quad$ Sin prótesis & 6 & & \\
Prótesis & & & \\
$\quad$ Incisión lateral externa & 167 & 28,2 & $20,5 \%$ \\
$\quad$ Técnica de Spira modificada & 242 & 40,8 & $22,3 \%$ \\
$\quad$ Patrón Wise corto & 82 & 13,8 & $17,1 \%$ \\
$\quad$ Incisión radial externa & 5 & 0,8 & $20 \%$ \\
\hline
\end{tabular}


Tabla 4 - Complicaciones y secuelas en los 3 grupos del estudio

\begin{tabular}{|c|c|c|c|}
\hline & $\begin{array}{l}\text { Grupo } 1 \\
\mathrm{~N}=125\end{array}$ & $\begin{array}{c}\text { Grupo } 2 \\
\mathrm{~N}=71\end{array}$ & $\begin{array}{l}\text { Grupo } 3 \\
\mathrm{~N}=100\end{array}$ \\
\hline \multicolumn{4}{|l|}{ Complicaciones } \\
\hline Necrosis cutánea & $8(6,4 \%)$ & $6(8,4 \%)$ & $7(7 \%)$ \\
\hline Infección & $6(4,8 \%)$ & $4(5,6 \%)$ & $5(5 \%)$ \\
\hline Seroma mantenido & $7(5,6 \%)$ & $6(8,4 \%)$ & $6(6 \%)$ \\
\hline Hematoma & $7(5.6 \%)$ & $2(2,8 \%)$ & $8(8 \%)$ \\
\hline Reintervención primer mes postoperatorio & $8(6,4 \%)$ & $3(4,2 \%)$ & $4(4 \%)$ \\
\hline \multicolumn{4}{|l|}{ Causas de reintervención por secuelas } \\
\hline Contractura capsular III/IV & $16(12,8 \%)$ & $9(12,6 \%)$ & $9(9 \%)$ \\
\hline Rotura implante & $8(6,4 \%)$ & $1(1,4 \%)$ & $6(6 \%)$ \\
\hline Reconstrucción CAP & $5(4 \%)$ & & \\
\hline Resección pliegues cutáneos & $5(4 \%)$ & & $4(4 \%)$ \\
\hline Extrusión protésica & $4(3,2 \%)$ & $1(1,4 \%)$ & \\
\hline Asimetría prótesis desplazada & $2(1,6 \%)$ & & $1(1 \%)$ \\
\hline Infección mantenida & $2(1,6 \%)$ & $1(1,4 \%)$ & $1(1 \%)$ \\
\hline
\end{tabular}

primer mes postoperatorio fue del $20 \%$ (20/100). Los resultados se exponen en la tabla 4.

\section{Discusión}

En los últimos años, asistimos a un aumento del número de pacientes en las que se realiza mastectomía como cirugía de rescate por recurrencia local tras cirugía conservadora y radioterapia, así como de aquellos casos en los que, en pacientes que van a precisar radioterapia postoperatoria por un estadio localmente avanzado, se realiza mastectomía preservadora de piel ${ }^{10}$. Es necesario, por tanto, conocer si la reconstrucción inmediata tiene una tasa de complicaciones aceptable en los escenarios descritos.

En general, las tasas publicadas de complicaciones precoces en las pacientes tratadas mediante mastectomía ahorradora de piel tras radiación previa o posterior a la reconstrucción son superiores a las de las pacientes no radiadas, pudiendo superar el $30 \%$ de los $\operatorname{casos}^{11,12}$. Por el contrario, las 3 cohortes de nuestro estudio experimentaron tasas de complicaciones en el postoperatorio inmediato similares (28,8\% en grupo 1, 29,6\% en grupo 2 y $30 \%$ en grupo 3 , con $p=0,21$ ), aunque el tamaño de nuestro estudio no es suficiente para establecer diferencias significativas y concluir que la radioterapia previa no influye en este hecho.

Encontramos en la literatura algunos estudios similares al nuestro en cuanto que comparan los resultados de la reconstrucción inmediata en cohortes de pacientes que han recibido previamente radioterapia mamaria o como tratamiento adyuvante.

Sbitany et al. ${ }^{5}$ centran su estudio en las complicaciones postoperatorias inmediatas, describiendo un aumento del riesgo relativo, similar en los 2 grupos de pacientes, para la aparición de complicaciones como hematoma, seroma, infección, necrosis de piel o CAP, dehiscencia de sutura o extrusión del implante. Al igual que ellos, en nuestro estudio encontramos que la tasa de necrosis e infección es superior en el grupo de pacientes que reciben radioterapia adyuvante, apareciendo seroma o hematoma con una frecuencia similar en ambos grupos de pacientes.
En el estudio publicado recientemente por Sosin et al. ${ }^{13}$ se analizan tanto complicaciones precoces como secuelas y necesidad de cirugía de revisión en las 2 cohortes de pacientes. En dicho estudio tanto la tasa de complicaciones preoperatorias precoces como el porcentaje de secuelas que requieren reintervenciones después del primer mes es superior en la cohorte de pacientes que recibe radioterapia adyuvante $(33,3 \%$ vs. $26,3 \%$ y $28,6 \%$ vs. $10,5 \%$, respectivamente). Por el contrario, nuestros hallazgos indican mayor predilección de secuelas en pacientes con radioterapia previa a la reconstrucción, especialmente en lo que atañe a complicaciones estéticas (fundamentalmente, contractura capsular), con una tasa de reintervención de casi el doble (33,6\% en grupo 1 vs. 16,9\% en grupo $2, p=0,067)$, siendo estadísticamente significativo. Al igual que Sosin et al., Spear et al. ${ }^{14}$ encuentran que la contractura capsular ocurre más frecuentemente en aquellas pacientes que reciben radioterapia adyuvante (40\% de los casos vs. 7,8\% de las pacientes con radiación previa).

Además de la radioterapia, se han descrito algunos predictores específicos de las complicaciones tras mastectomía ahorradora de piel y pezón, como son un índice de masa corporal elevado, el tabaquismo activo y o el tipo de incisión realizada, siendo utilización la periareolar aquella asociada a mayor riesgo de necrosis del $\mathrm{CAP}^{15}$. No hemos analizado el peso de estos factores en la aparición de complicaciones, si bien hemos observado que las 3 cohortes son equiparables en cuanto a la presencia de estos factores de riesgo. Si analizamos la técnica quirúrgica empleada, en nuestra serie se han observado tasas de complicación similares a las publicadas en la literatura para las diferentes técnicas, como se muestra en la tabla 2.

Todos los trabajos consultados concluyen que si bien la radioterapia, ya sea previa ${ }^{10,16}$ o posterior a la mastectomía ${ }^{17}$, es causa de un mayor número de complicaciones, estas no contraindican la realización de reconstrucción inmediata, justificado por unos adecuados resultados estéticos y a la satisfacción de las pacientes, aspectos que nosotros no hemos analizado en nuestro estudio.

Nuestros hallazgos identifican la cohorte de pacientes tratadas mediante radioterapia previamente a mastectomía con reconstrucción inmediata como más propensas para 
desarrollar una complicación a largo plazo/secuela y requerir reintervención que las pacientes que reciben la radioterapia después o las que no son tratadas mediante radiación.

Sin embargo, existen algunas limitaciones en este estudio, al tratarse de la experiencia de una institución única con un número de casos relativamente pequeño, por lo que no podemos generalizar nuestros resultados.

En conclusión, la tasa de complicaciones a largo plazo y la tasa de reintervenciones son mayores en el grupo MB + RMI con radioterapia previa que en los grupos $\mathrm{MB}+\mathrm{RMI}$ con radioterapia posterior $\mathrm{O} M B+\mathrm{RMI}$ sin radioterapia.

\section{Conflicto de intereses}

Los autores declaran no tener ningún conflicto de intereses.

\section{B I B L I O G R A F Í A}

1. Berry MG, Gomez KF. Surgical techniques in breast cancer: An overview. Surgery (Oxford). 2013;31:32-6.

2. American Cancer Society. Breast cancer facts \& figures 20132014. American Cancer Society, Atlanta, 2013.

3. Kummerow KL, Du L, Penson DF, Shyr Y, Hooks MA. Nationwide trends in mastectomy for early-stage breast cancer. JAMA Surg. 2015;150:9-16.

4. Clemens M, Steve K. Current perspectives on radiation therapy in autologous and prosthetic breast reconstruction. Gland Surg. 2015;4:222-31.

5. Sbitany H, Wang F, Peled AW, et al. Immediate implantbased breast reconstruction following total skin-sparing mastectomy: Defining the risk of preoperative and postoperative radiation therapy for surgical outcomes. Plast Reconstr Surg. 2014;134:396-404.

6. Tang R, Coopey S, Colwell A, et al. Nipple-sparing mastectomy in irradiated breasts: Selecting patients to minimize complications. Ann Surg Oncol. 2015;22:3331-7.
7. Recht A, et al. Postmastectomy radiotherapy: Clinical practice guidelines of the American Society of Clinical Oncology.American Society of Clinical Oncology. J Clin Oncol. 2001;19:1539-69.

8. Bartelink $\mathrm{H}$, et al. Impact of a higher radiation dose on local control and survival in breast-conserving therapy of early breast cancer: 10-year results of the randomized boost versus no boost EORTC 22881-10882 trial. J Clin Oncol. 2007;25:3259-65.

9. NCCN Guidelines-v.2. 2013. Consenso de BT en Ca mama 2010 de la SEOR.

10. Colwell A, Tessler O, Lin A, et al. Breast reconstruction following nipple-sparing mastectomy. Plast Reconstr Surg. 2014;133:496-506

11. Vandeweyer E, Deraemaecker R. Radiation therapy after immediate breast reconstruction with implants. Plast Reconstr Surg. 2000;106:56-8.

12. Foster RD, Esserman LJ, Anthony JP, Hwang ES, Do H. Skin-sparing mastectomy and immediate breast reconstruction: A prospective cohort study for the treatment of advanced stages of breast carcinoma. Ann Surg Oncol. 2002;9:462-6.

13. Sosin $\mathrm{M}$, et al. Timing of radiation therapy in nipple-sparing mastectomy influences outcomes and patient-reported quality of life. Breast J. 2018.

14. Spear S, Shuck J, Hannan L, Albino F, Patel K. Evaluating long-term outcomes following nipple-sparing mastectomy and reconstruction in the irradiated breast. Plast Reconstr Surg. 2014;133:605e-14e.

15. Endara M, Chen D, Verma K, Nahabedian MY, Spear SL. Breast reconstruction following nipple-sparing mastectomy: A systematic review of the literature with pooled analysis. Plast Reconstr Surg. 2013;132:1043-54.

16. Cordeiro PG, et al. Inmediate tissue expander/implant brast reconstruction after Salvage Mastectomy for cancer recurrence following lumpectomy/irradiation. Plast Reconstr Surg. 2012;129:341-50.

17. Cordeiro. et al. Irradiation after immediate tissue expander/ implant breast reconstruction: Outcomes, complications, aesthetic results, and satisfaction among156 patients. Plast Reconstr Surg. 2004;113:877-81. 